\title{
SINAIS INTERNACIONAIS E A FORMAÇÃO PARA INTÉRPRETES DE SINAIS INTERNACIONAIS
}

\author{
INTERNATIONAL SIGN AND TRAINING FOR INTERNATIONAL SIGN \\ INTERPRETERS
}

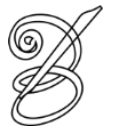 \\ Letícia Fernandes Garcia Wagatsuma GRANADO \\ Universidade Federal de Santa Catarina
}

\begin{abstract}
Resumo: Sinais Internacionais (SI) não é considerado como uma língua oficialmente, pois ele não tem uma comunidade de origem definida. Entretanto, é muito bem aproveitado como uma espécie de língua franca equidistante das diversas línguas de sinais de cada país, em encontros internacionais de surdos. SI não pode se comparar com o Esperanto, como uma língua não planejada e, também, não pode se definir como Gestuno, que é referenciado aos gestos, mas pode ser usado em encontros internacionais. A partir de 1987, começou a interpretação de Sinais Internacionais no Congresso da Federação Mundial de Surdos (WFD), e desde então, vem se desenvolvendo. A Associação Mundial de Intérpretes de Língua de Sinais (WASLI) oferece um credenciamento provisório para os intérpretes que têm experiência da interpretação de SI de no mínimo três anos. O surdo pode atuar como um intérprete profissional tanto quanto os ouvintes. Os intérpretes surdos passaram a ser reconhecidos na comunidade surda a partir de 1993, quando iniciaram sua atuação nas conferências (CAMPELLO, 2014) realizadas no Brasil, onde ainda não havia formação específica na interpretação para os surdos. Por esta razão, precisamos fomentar mais pesquisas e investir na qualificação e na formação desses profissionais.
\end{abstract}

Palavras-chave: Sinais Internacionais. Interpretação de Sinais Internacionais. Intérprete Surdo. Formação.

Abstract: International Sign (IS) is not officially considered a language, as it does not have a defined community of origin. However, it is very well used as kind of equidistant lingua franca from different sign languages of each country, in international deaf meetings. IS cannot be compared with Esperanto as it is not a planned language and also cannot be defined as "Gestuno", which refers to gestures. From 1987, the interpreting of International Sign began at the World Federation of the Deaf Congress (WFD), and has been developing to date. The World Association of Sign Language Interpreters (WASLI) provides interim accreditation for interpreters who have experience in IS interpreting for at least 3 years. Deaf people can act as a professional interpreters as much as the hearing. Deaf interpreters began to be recognized in the Brazilian deaf community from in 1993, when they started to work at international conferences (CAMPELLO, 2014) in Brazil, where there is still no specific training for deaf interpreters. To this end, we need to encourage more research and invest in qualification and training of these professionals.

Keywords: International Sign. International Sign Interpreting. Deaf Interpreter. Training.

RECEBIDO EM: 14 de março de 2018

ACEITO EM: 11 de dezembro de 2018

PUBLICADO EM: janeiro 2019 
Figura 1 - Termos e autores tratados neste artigo

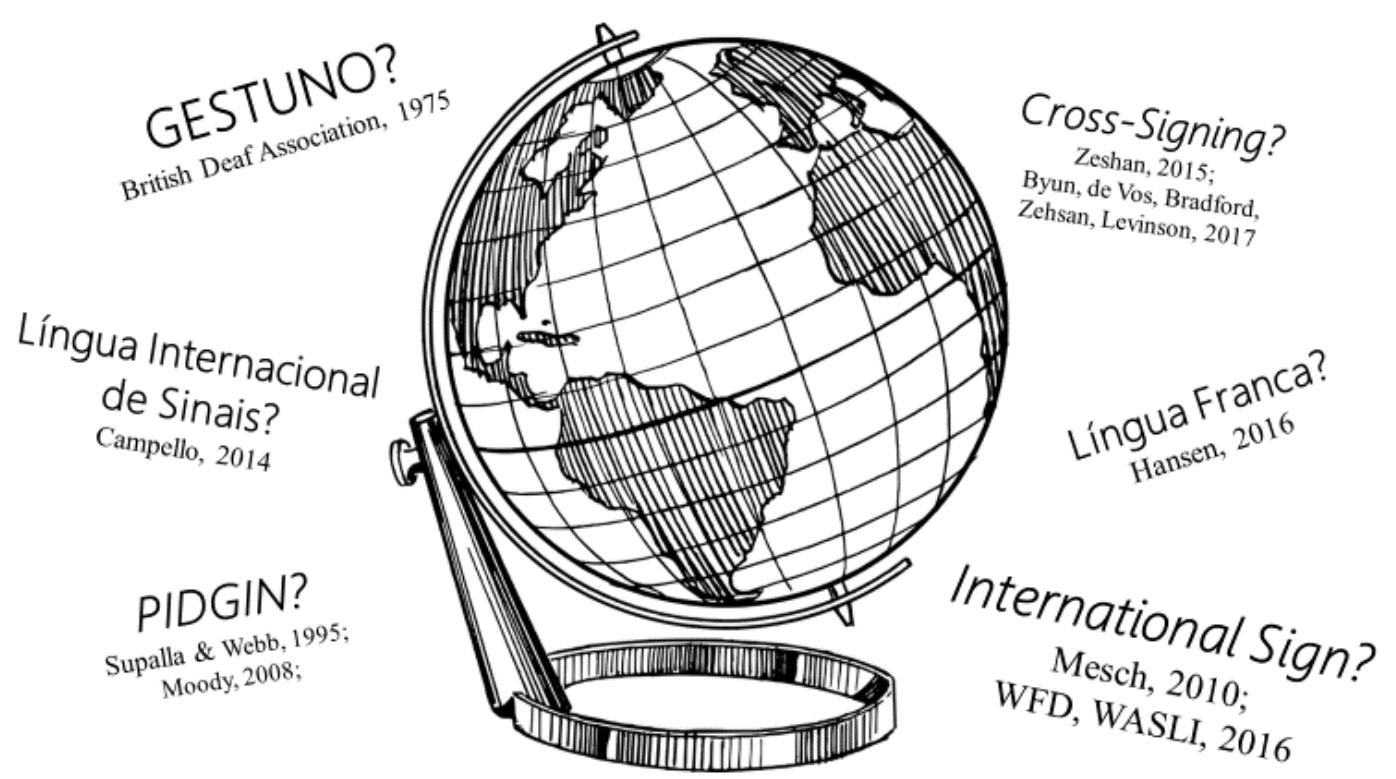

Elaboração: Letícia Fernandes Garcia Wagatsuma

\section{Conhecendo o termo: Sinais Internacionais}

2127 nternational Sign (IS)? É uma língua? Campello (2014, p. 147) define o termo "Língua de Sinais Internacionais" como "um sistema de sinais internacionais com o objetivo de melhor entendimento o uso de várias línguas de sinais, para criar uma língua fácil de aprender e de se comunicar". Mas o termo "Língua de Sinais Internacionais" não é mais usado: a World Federation of the Deaf (WFD) votou contra a proposta para reconhecer Sinais Internacionais como uma língua, o que aconteceu provavelmente porque os pesquisadores em linguística e usuários de Sinais Internacionais não aprovaram essa ideia (MOODY, 2008, p. 26). Mesch (2010, p. 4) também acrescenta que atualmente o termo "International Sign é preferido, porque SI pode variar dependendo do contexto linguístico dos sinalizadores que o utilizam" ${ }^{1}$. Esse contexto pode variar de língua, cultura, história dos sinais de seu país de origem. "O uso do termo International Sign, ao invés de International Sign Language, enfatiza que International Sign não é reconhecido como um status linguístico completo" 2 (ADAM, 2012, p. 853). No Brasil, adotamos o termo "Sinais Internacionais", em português, amplamente utilizado pela comunidade surda. Sinais Internacionais não é considerado como uma língua oficialmente, pois ele não tem uma comunidade de origem definida. Mesmo assim, serve como uma espécie de língua franca equidistante em eventos e encontros internacionais de surdos e é convencional para os membros da WFD. Ele "passou a ser usado sistematicamente" (CAMPELLO, 2014, p. 147) não só por ser convencional, mas também por ser uma 
“comunicação visual entre pessoas surdas de países diferentes que não compartilham uma única língua de sinais" ${ }^{3}$ (CRASBORN; HIDDINGA, 2011, p. 492). Assim, quando um surdo de um país se encontra com um surdo estrangeiro, eles se comunicam expressando-se espontaneamente em SI. Mesch (2010) distingue as duas diferenças em uso de SI na comunicação: SI convencionalizado e comunicação informal entre os usuários das línguas de sinais nacionais. Sinais Internacionais convencionalizado é mais usado nos eventos e reuniões internacionais, enquanto o SI informal tem sido usado durante encontros informais de pessoas surdas nas viagens. Quando um surdo encontra um surdo de outro país, ambos ainda não sabem SI. Como se comunicam? Quando os usuários de língua de sinais nunca tiveram contato em SI antes, isso pode ser chamado de cross-signing $^{4}$ (ZESHAN, 2015), quando pela primeira vez espontaneamente surge a comunicação entre eles. Os usuários surdos de língua de sinais de países diferentes que não compartilham uma língua comum, sendo sinalizada ou escrita, são facilmente capazes de se engajar em conversas, por exemplo, durante eventos internacionais ou quando viajam (BYUN et al., 2017).

Moody (2008, p. 20, tradução minha) evidencia que "SI tem sido usado pelos surdos europeus há 250 anos no mínimo, mas a comunicação com os surdos que vieram de outros países estava tendo sucesso nos encontros europeus vários séculos antes disso" 5 . Adam (2012, p. 854) relata que SI é um modo eficaz de comunicação para muitas pessoas surdas em contextos transnacionais e tem sido utilizado como uma língua franca em eventos como a Deaflypmics 6 desde o seu início, com os primeiros "Jogos Silenciosos” em 1924, em que nove países europeus participaram. No ano de 1950, a WFD “considerou desejável alguma padronização de 'gestos internacionais' e foi formado um comitê de 'Unificação de Sinais"” 7 (MOODY, 2008, p. 24) para selecionar os "sinais mais naturais, espontâneos e fáceis de uso comum por surdos de diferentes países" 8 (BDA, 1975). Em 1975, a Associação de Surdos Britânicos (BDA) publicou na Inglaterra o livro "Gestuno: International Sign Language of the Deaf”, que tem mais de 1.500 vocabulários de 'sinais internacionais', mas "os surdos começaram a reclamar que os sinais no Gestuno não eram suficientemente icônicos para serem facilmente compreendidos" 9 (MOODY, 2008, p. 24). Rosenstock e Napier (2016, p. 4) definem o conceito Gestuno como sendo "referenciado aos gestos e ao senso de unidade" ${ }^{10}$. Em 1976, o Gestuno foi usado pela primeira vez no congresso da WFD na Bulgária, e era incompreensível para os participantes surdos (MOODY, 2008). Baker-Shenk e Cokely (1991) afirmam que o Gestuno é como o Esperanto, que não pode ser considerado uma língua por várias razões: (1) não possui gramática própria; (2) não existem usuários nativos do Gestuno; (3) pouquíssimas pessoas são fluentes 
nesta modalidade de comunicação, somente quando há oportunidade de usar ou praticar. Logo depois disso, o Gestuno foi descartado e Moody começou a usar SI, que aprendeu com as pessoas surdas durante as viagens.

Desde que distinguiram que o uso do Gestuno na comunicação e na interpretação não obteve nenhum sucesso, este termo "ficou fora de uso, e o livro também ficou mal-visto" 11 (ROSENSTOCK; NAPIER, 2016, p. 4). A tentativa de aplicar o Gestuno resultou em um desastre comunicativo, porque os intérpretes não fizeram o uso do espaço, da ação construída ou de expressão facial (HANSEN, 2016). Por essa razão esta modalidade ficou escassa e caiu em desuso. Lembrando, mesmo que seja usado para comunicação entre os limites de línguas de sinais diferentes e tendo empréstimos de várias línguas de sinais, SI não pode se comparar com o Esperanto porque não é uma língua planejada, com um léxico estabelecido e um conjunto fixo de regras gramaticais (ADAM, 2012, p. 853). O termo "Sinais Internacionais" ainda é discutido em poucos estudos (publicados): Sinais Internacionais "também pode ser a língua franca para quebrar as barreiras linguísticas" 12 (HANSEN, 2016, p. 21) em uso nas conferências internacionais. Hansen (2016, p. 15) complementa que SI não é o tipo da língua universal,

214 "entendida da mesma maneira e na mesma medida por sinalizadores em todo o mundo" ${ }^{13}$. Para Moody (2008), Supalla e Webb (1995) e McKee e Napier (2002), Sinais Internacionais se define como pidgin. Moody (2008) considera pidgin como uma língua de contato, com uma mistura de duas ou mais línguas em contato, em situações específicas. Ele considera o termo SI como pidgin em razão de ser uma língua de contato que evoluiu entre as línguas de sinais. Supalla e Webb (1995) também definem este termo como pidgin típico, em uso de contato com grupos de línguas diferentes em algumas ocasiões específicas. Contudo, a partir dos resultados das suas pesquisas, eles descrevem outro tipo de pidgin em uso de Sinais Internacionais que é chamado de koine, o qual "se desenvolve entre as línguas faladas, onde as línguas em contato são extremamente semelhantes" ${ }^{14}$ (HOLM, 1988; ROMAINE, 1988 apud SUPALLA; WEBB, 1995, p. 349). O termo pidgin não se aplica muito bem ao SI. De acordo com Hansen (2016), cada pidgin pode diferir estruturalmente de acordo com os fatores linguísticos, sociais e históricos.

Os estudos sobre o pidgin desenvolveram-se fortemente nas línguas europeias em contextos (pós-)coloniais durante as duas últimas décadas. Whynot (2016, p. 35) descreve este termo da seguinte forma: "SI é uma área não suficientemente pesquisada, o contato de línguas de sinais que serve como uma língua franca de fato para a globalização de comunidades surdas" 15. Para Crasborn e Hiddinga (2011), o termo SI refere-se às formas de contato espontâneo em 
contraste com formas mais convencionalizadas. Para lembrarmos, com o uso de diferentes elementos icônicos não convencionais, SI é bastante diferente das línguas de sinais nacionais que possuem um léxico padronizado e uma gramática fixa (HANSEN, 2016), não sendo uma língua universal. Hansen explica o motivo do SI não se enquadrar no conceito:

Não é uma língua nativa de uma comunidade surda, transmitida dos pais para filhos e de colegas para colegas. É usado somente em momentos específicos e em posições variadas, quando os usuários de diferentes línguas de sinais se reúnem. ${ }^{16}$ (HANSEN, 2016, p. 17).

Resumindo, referente às distintas perspectivas dos autores mencionados sobre o termo "Sinais Internacionais", os conceitos e os argumentos embasados, pode ser considerado basicamente uma língua franca, com uma mistura de diversas línguas de sinais nacionais. Em consequência disso, é convencionado ad hoc, por exemplo, em eventos, e expressado espontaneamente pelos surdos em encontros e conferências internacionais.

\section{Formação para Intérpretes de Sinais Internacionais}

A formação profissional em uma área específica da interpretação em International Sign ainda não existe por não ser uma língua oficial, mas é "amplamente utilizado em reuniões internacionais onde os participantes não compartilham uma língua de sinais em comum" 17 (EUD, 2012 apud WIT; SLUIS, 2016, p. 108). Essas autoras dizem que o status linguístico do SI é vigorosamente debatido. Devido à falta de uma formação profissional, os intérpretes do SI não são oficialmente registrados e não possuem reconhecimento oficial como profissional (WIT; SLUIS, 2016, p. 110). Mesmo assim, as associações internacionais com apoio em todo o mundo, tais como o EFSLI ${ }^{18}$ (European Forum of Sign Language Interpreters), a EUD ${ }^{19}$ (European Union of the Deaf), a WASLI ${ }^{20}$ (World Association of Sign Language Interpreters) e a WFD ${ }^{21}$ (World Federation of the Deaf) incentivaram-se a realização do reconhecimento formal, criando um registro para os intérpretes de International Sign (WIT; SLUIS, 2016, p. 110).

$\mathrm{Na}$ Europa, existe uma pós-graduação internacional que visa contribuir para o desenvolvimento do campo profissional de interpretação entre pessoas surdas e ouvintes, chamada de EUMASLI ${ }^{22}$ (European Master in Sign Language Interpreting). Esse programa é uma colaboração entre três universidades: 1) Magdeburg-Stendal University of Applied Science (Alemanha); 2) Humak University of Applied Sciences (Helsinque e Kuopio, Finlândia); e 3) Heriot-Watt University (Edimburgo, Escócia). O programa tem sido oferecido para um grupo 
internacional dos intérpretes de língua de sinais desde 2009 (HESSMANN et al., 2011). Essa pós-graduação oferece os seguintes módulos para desenvolver a formação: 1) habilidades internacionais; 2) desenvolvimento da profissão; 3) desenvolvimento da pesquisa. Com esses módulos, oferecem a aplicação e o fortalecimento das competências linguísticas e tradutórias, promovendo conhecimentos sociais e políticos do trabalho como intérprete profissional, preparação necessária à formação profissional (HESSMANN et al., 2011). Nessa pósgraduação, há cinco semestres com disciplinas para os estudos de interpretação e tradução. No seu currículo, possui algumas disciplinas relacionadas ao Sinais Internacionais: 1) IS: Introducing International Sign; 2) Translating between English and International Sign; 3) Interpreting between English and International Sign; e outras disciplinas.

Nesse caso, o intérprete que tiver interesse em interpretação de SI poderia também conhecer o trabalho e os objetivos da WASLI. Um desses objetivos é promover a profissão de interpretação de língua de sinais em todo o mundo, e também apoiar as pessoas surdas para que tenham a possibilidade de treinar e atuar como intérpretes. Em 1987, no Congresso da WFD, começou a interpretação de SI, que tem sido desenvolvida até hoje. A WASLI (WFD-WASLI 216 International, 2016) define o conceito SI como uma variedade de contato de línguas de sinais usada em uma variedade de contextos diferentes, particularmente em reuniões internacionais tais como a WFD, eventos como o Deaflypmics, vídeos-clipes produzidos por surdos e assistidos por outras pessoas surdas de todo o mundo. Por isso, Aquiline (2006) sugere que a WASLI e a WFD devem: 1) criar um currículo de formação para os intérpretes; 2) conduzir a avaliação e a certificação de qualificação dos intérpretes de SI; 3) criar os padrões da prática para intérpretes de SI; e 4) manter um registro dos intérpretes de SI certificados para as conferências internacionais (AQUILINE, 2006 apud SHENEMAN; COLLINS, 2016).

A WASLI possui um sistema de credenciamento que visa a definir, manter e promover as normas em interpretação de SI, conforme com a Política e as Diretrizes Provisórias de Credenciamento. Esse sistema realiza anualmente bancas para avaliar e certificar os intérpretes de SI, para garantir o seu trabalho e a sua qualidade profissional. O credenciamento, que vale por cinco anos, também visa a manter um sistema de desenvolvimento profissional ao longo da vida. Os requisitos exigidas pela WASLI (WFD-WASLI, 2016) para se qualificar como um profissional são: 1) possuir um conhecimento sobre assuntos atuais, por exemplo, geografia internacional, história, cultura e assuntos sociais; 2) possuir uma familiaridade com a história e a estrutura organizacional internacional de surdos e intérpretes de língua de sinais tais como a da WFD, da EUD, da WASLI, do EFSLI e da Deaflympics; 3) possuir um conhecimento das 
instituições internacionais relacionadas e em cooperação com as organizações de Intérpretes de Língua de Sinais, por exemplo, a Organização das Nações Unidas (ONU), a Aliança Internacional de Deficiência (IDA), a Comissão Europeia, o Parlamento Europeu, o Fórum Europeu da Deficiência, bem como conhecimento da geografia internacional, história, cultura, e outros assuntos sociais; 4) possuir um conhecimento dos países nacionais, da sua cultura, política e história. Além de adquirir todos esses conhecimentos, os intérpretes de SI também precisam saber sobre a qualificação do trabalho, o que inclui: 1) reconhecimento formal no órgão de registro profissional de intérpretes de línguas de sinais; 2) um diploma universitário, preferencialmente em um campo relevante, por exemplo, interpretação e/ou estudos de tradução, interpretação de língua de sinais (por exemplo, bacharelado em Letras-Libras, no Brasil); 3) participação em organizações nacionais e internacionais de intérpretes profissionais, por exemplo, a WASLI, o EFSLI, a AIIC ${ }^{23}$ (no Brasil, por exemplo, a FEBRAPILS, a FENEIS ${ }^{24}$ nacional e regional e as associações nacionais de surdos); 4) participação nas organizações regionais, nacionais e internacionais de surdos e intérpretes de língua de sinais, tais como a WFD e a EUD (Ex.: FENEIS).

Moody (2008) apresenta os três requisitos básicos para aprender Sinais Internacionais antes de tornar-se um intérprete de SI: 1) para se tornar fluente em SI, o intérprete deve ser fluente em pelo menos uma língua de sinais nacional, mas é preferível conhecer duas a quatro línguas de sinais nacionais. Quanto mais conhecimento em línguas de sinais, maior flexibilidade ele terá para se adaptar ao vocabulário em uso em locais e contextos diferentes; 2) ter experiência de comunicação com pessoas surdas em diferentes países, ampliando o conhecimento da cultura, política, família, religião, educação, trabalho e diversão durante as viagens extensivas. Assim, ele pode selecionar os sinais mais icônicos para criar as imagens visuais, que será mais compreensível; 3) é preciso ter uma mente aberta e flexível, bem como o desejo e a capacidade de cultivar a criatividade na expressão.

Além de conhecer várias línguas de sinais nacionais e culturas adicionais, "o intérprete precisa ter uma variedade de estratégias de interpretação flexíveis para responder às exigências únicas em um ambiente multilíngue e multicultural" 25 (SHENEMAN; COLLINS, 2016, p. 171), e a competência intercultural é uma habilidade essencial para um intérprete que trabalha num ambiente multilíngue e multicultural (WIT, 2010 apud SHENEMAN; COLLINS, 2016). Em seguida, Wit (2010) identifica as três razões pelas quais a interpretação em contextos internacionais multilíngues é ainda mais desafiadora: 
Primeiro, como mencionado anteriormente, intérpretes geralmente são treinados em duas línguas e culturas. Trabalhar em contextos internacionais expõe os diferentes níveis de fluências em várias línguas usadas no evento [...]. Em seguida, as complexidades adicionais afetam a capacidade de processamento cognitivo do intérprete. Terceiro, o intérprete é encarregado de trabalhar com os usuários nãonativos de ambos os idiomas em que está treinado. ${ }^{26}$ (WIT, 2010 apud SHENEMAN; COLLINS, 2016, p. 172).

O intérprete, apesar de adquirir as experiências em contexto de conferência internacional, precisa conhecer as estratégias para usar a flexibilidade e a criatividade na interpretação de SI para um público de diversas línguas de sinais nacionais e diversas culturas. A interpretação de SI não deve ser traduzida sinal por sinal, por ser flexível com uma variedade de contato de línguas de sinais, no caso da interpretação intramodal. O objetivo da interpretação de SI é fornecer uma equivalência através da interpretação livre (SHENEMAN; COLLINS, 2016). No caso da interpretação intermodal inglês-SI, McKee e Napier definem a equivalência:

A definição de equivalência é central para o conceito de interpretação livre, e afirmamos que, no contexto da interpretação de Sinais Internacionais, a equivalência é procurada pelo intérprete que infere o significado com base em sua compreensão da situação e do texto da linguagem fonte e predizendo o que o texto é provável para significar para o público-alvo. ${ }^{27}$ (MCKEE; NAPIER, 2002, p. 31).

$\mathrm{Na}$ interpretação livre, a estrutura linguística da língua-fonte é ignorada e um equivalente é encontrado com base no significado que transmite (CRYSTAL, 1987 apud MCKEE; NAPIER, 2002, p. 31). No caso da interpretação de SI, McKee e Napier (2002) explicam que o objetivo principal é recriar uma aproximação da mensagem essencial em uma linguagem híbrida, que se forma em elementos tirados de várias línguas e que, por vezes, pode exigir alguma redução de conteúdo.

\section{Intérpretes surdos}

Atualmente, no Brasil, os intérpretes ouvintes são encontrados em contexto escolar, acadêmico e religioso. Eles têm certificação de proficiência em Tradução e Interpretação de Libras $\left(\right.$ ProLibras $^{28}$ ) e também possuem conhecimento na área de interpretação e tradução, que adquiriram no bacharelado do curso de Letras-Libras. Segundo Rodrigues e Beer (2015, p. 31), “os primeiros intérpretes de língua de sinais de que se têm registros eram práticos, sem nenhuma formação acadêmica. Na maioria dos casos, eram familiares e amigos dos surdos ou religiosos preocupados em oferecer assistência ou evangeliza-los". Atualmente, os intérpretes de Libras são encontrados nas universidades, escolas, igrejas, prefeituras e outros lugares. Os intérpretes 
também podem trabalhar autonomamente. Os surdos podem solicitar intérpretes particulares para acompanhá-los em consultas médicas, reuniões de trabalho, na justiça e em outras situações.

Mas o surdo pode atuar como um intérprete? Boudreault (2005) coloca a questão no seu capítulo: "Como uma pessoa surda pode ser um intérprete de língua de sinais na sua própria comunidade surda? Isso não pode ser. Você é surdo!" ${ }^{29}$ (BOUDREAULT, 2005, p. 323). Afinal, o que é o "intérprete surdo"? Apresenta-se a definição deste conceito no site Deaf Interpreter Institute:

Um Intérprete para Surdos é um especialista que presta serviços de interpretação, tradução e transliteração na Língua de Sinais Americana e outras formas de comunicação visuais e táteis usadas por pessoas que são surdas, deficientes auditivas e surdo-cegas 30 .

Essa questão tem sido considerada por surdos e ouvintes. É muito comum encontrar intérpretes que ouvem na comunidade surda. Estes trabalham com a interpretação consecutiva, e na maioria das situações, com a interpretação simultânea de Libras para Português ou viceversa. Por isso, os intérpretes surdos podem ser confundidos com os intérpretes ouvintes. O intérprete surdo é praticamente um assunto novo para a comunidade surda. "O uso profissional de intérpretes surdos está aumentando em vários países e em diversos contextos" ${ }^{31}$ (STONE; RUSSELL, 2011, p. 100). Essa nova tendência tem surgido naturalmente na comunidade surda, surgem novas pesquisas sobre o intérprete surdo no mundo todo e o desenvolvimento da atuação dos intérpretes surdos brasileiros ainda está crescendo. No Brasil, os intérpretes surdos da modalidade interlingual passaram a ser reconhecidos a partir de 1993 (CAMPELLO, 2014), quando iniciaram sua atuação no espaço acadêmico. Reis (2013) elucida a história dos intérpretes surdos: eles já existiam muitos anos atrás, antes do congresso de Milão em 1880, com suas diversas experiências de tradução (tradução de sinais caseiros; gestos e tradução de escrita para língua de sinais). Embora não houvéssemos percebido, os intérpretes surdos sempre estiveram ali para ajudar outros surdos. Boudreault (2005, p. 324) afirma que

[h]á muitas possibilidades de interpretação informal dentro da comunidade surda, onde alguns membros da comunidade possuem numerosas habilidades para atuar como facilitadores de comunicação. O contexto pode ser dentro de uma escola de surdos, no local de trabalho ou, ao se encontrar com pessoas ouvintes profissionais como advogados, médicos etc. ${ }^{32}$ 
Mas até hoje, a questão "o surdo pode atuar como intérprete?" permanece entre as pessoas na comunidade surda. A atuação do intérprete surdo ainda está se desenvolvendo no campo de interpretação e tradução no Brasil. Boudreault (2005, p. 324) afirma que o processo do intérprete surdo pode envolver várias ações tais como expressar, gesticular, escrever, ou usar outras línguas de sinais. Esses processos da interpretação são vários, e cada intérprete surdo tem a própria experiência na prática; depende da situação em que ele pode se sentir seguro para trabalhar.

Essa diferença entre intérprete surdo e intérprete ouvinte ainda pode ser discutida no campo profissional. Essa nova perspectiva pode ser formulada nos seguintes termos: "como Intérpretes/Tradutores Surdos definiram o trabalho, e como eles se definiram como intérprete" 33 (STONE, 2009, p. 80). Com essa reflexão, o autor também argumenta que "estas diferenças podem identificar se o intérprete/tradutor surdo empreende o mesmo trabalho ou um trabalho diferente, ou definir o papel de um Intérprete 'Surdo' como diferente do intérprete 'ouvinte'” 34 (STONE, 2009, p. 80). Existem alguns aspectos que diferenciam a forma de interpretação no campo profissional entre intérpretes ouvintes e intérpretes surdos, por eles possuírem diferentes relações com a cultura surda. Os ouvintes, por possuírem a melhor compreensão e eficiência em língua falada, "usam a audição como funcionamento auditivo pela habilidade nos atos do ouvir e do falar" (CAMPELLO, 2014, p. 148). Quanto aos surdos, Campello compara a diferença destes com os ouvintes, que "acontece o mesmo com as pessoas surdas que usam as mãos como funcionamento visual pela habilidade nos atos do ver e do sinalizar" (CAMPELLO, 2014, p. 148). Portanto, os surdos se expressam melhor usando a língua de sinais, isso é, “envolvendo o corpo todo, no ato da comunicação" (CAMPELLO, 2014, p. 148). Ela também acrescenta que a "sua comunicação é do viso-gestual e produz inúmeras formas de apreensão, interpretação e narração do mundo a partir de uma cultura visual" (CAMPELLO, 2014, p. 148). Perlin e Miranda (2003, p. 218) assim definem a cultura surda:

[...] experiência visual significa usar a visão [...] como meio de comunicação. Desta experiência visual surge a cultura surda representada pela língua de sinais, pelo modo de ser, de se expressar, de conhecer o mundo, de entrar nas artes, no conhecimento científico e acadêmico.

Por essa razão, alguns surdos exprimem possibilidade de atuarem como intérpretes por terem uma experiência visual e por conseguirem se expressar melhor usando a língua de sinais na comunicação com outros surdos. No caso da proficiência interpretativa, vale ressaltar que, além de serem nativos na língua de sinais, nas suas primeiras atuações como intérpretes, os 
surdos podem cometer os mesmos erros de intérpretes ouvintes iniciantes. Conforme os autores Adam et al. (2014), podemos apresentar algumas diferenças que eles incluem sobre os códigos de conduta na interpretação entre os intérpretes surdos e os intérpretes ouvintes:

1) Intérpretes surdos e ouvintes estão situados de forma diferente em relação aos seus hábitos (Bourdieu, 1991) (ex.: onde os intérpretes surdos e ouvintes estão culturalmente colocados dentro de uma comunidade de uma língua minoritária);

2) Intérpretes surdos e ouvintes interpretam diferentes nuances da língua de forma diferente. (ex.: intérpretes de surdos têm uma melhor compreensão das nuances de língua de sinais, e intérpretes ouvintes têm uma melhor compreensão das nuances de língua oral);

3) A fala não é sempre uma parte central do trabalho do intérprete surdo ${ }^{35}$ (ADAM et al., 2014, p. 7)

Complementando as diferenças entre eles, Morgan e Adam (2012 apud ADAM et al. 2014, p. 7) afirmam que a "aceitação e reconhecimento do inventário de língua do intérprete, conjunto de habilidades, qualificações e experiência também diferem entre intérpretes surdos e intérpretes ouvintes" ${ }^{36}$. Stone $(2009$, p. 80) apresenta que uma das principais diferenças é o nível de relevância para o público construído, pois “o INTÉRPRETE tende a representar aos intérpretes ouvintes e a VISÃO DO SURDO ['deaf in-vision' em tradução livre] representa aos tradutores/intérpretes surdos" ${ }^{37}$. Os intérpretes ouvintes e os intérpretes surdos têm diferentes focos no processo de interpretação (FORESTAL et al., 2012). Os intérpretes ouvintes concentram-se em assegurar que os consumidores ouvintes estão seguindo o processo, enquanto os intérpretes surdos se concentram, nos consumidores surdos.

Consequentemente, é importante distinguir as diferenças do trabalho entre os intérpretes surdos e intérpretes ouvintes e, também, incluir o intérprete surdo no campo de tradução e interpretação de Libras para reconhecer a sua competência profissional. Os intérpretes surdos devem se juntar aos intérpretes ouvintes no trabalho em equipe, no caso de conferências grandes.

O intérprete surdo, em vez de trabalhar com a interpretação interlingual, que interpreta uma língua de sinais para outra língua de sinais, também pode ser um intérprete intralingual, que traduz dentro de uma língua. É "uma interpretação de signos verbais por meio de outros signos da mesma língua" (JAKOBSON, 1992 apud CAMPELLO, 2014, p. 154). Segundo Luchi (2013, p. 41), "nas línguas de sinais há duas formas de produção de significado, uma pelas Estruturas Altamente Icônicas (EAI) e a outra pelo léxico padrão e apontamentos manuais". Na interpretação intralingual, a Libras Acadêmica pode ser traduzida para Libras usando as EAI, ou seja, as descrições imagéticas, para esclarecer o contexto da mensagem. 
Resumindo os dois conceitos, a interpretação interlingual é quando se tem duas línguas diferentes, e a intralingual é quando se tem apenas uma língua, que se traduz dentro de apenas uma língua. O intérprete surdo, além de ser um intérprete interlingual, é, segundo Pereira (2008), também um intérprete intralingual:

No caso em que uma pessoa surda não é falante competente de Libras e um ILS não consegue estabelecer um entendimento com ela, pode ser chamada outra pessoa surda que por meio gestual consiga uma comunicação primária, mas satisfatória e não se trata de uma interpretação interlíngue e sim de uma comunicação gestual ou mímica (PEREIRA, 2008, p. 143).

Os intérpretes surdos são qualificados no trabalho interlingual e são geralmente capazes de fazer a interpretação intralingual e vice-versa (ADAM et al., 2014) desde que seja em uma única modalidade espaço-visual (intramodal).

Os intérpretes surdos estão sendo cada vez mais reconhecidos na comunidade surda, revelando a grande importância de incluí-los no campo de tradução e interpretação de Língua de Sinais. Stone e Russell (2011) afirmam que veem grandes oportunidades de trabalho para 222 os intérpretes surdos que fornecem a interpretação de plataforma em conferências internacionais, ou fornecem a interpretação em transmissões de notícia na televisão.

\section{Conclusão}

Sinais Internacionais, considerado uma língua franca, tem sido muito usado pelo mundo todo, em eventos internacionais e viagens. Isso tem despertado o interesse e a curiosidade no Brasil, conquistando o espaço acadêmico e a comunidade surda. Aprendemos novos conteúdos com pesquisadores estrangeiros. Para isso, os intérpretes que têm interesse em transmitir os conteúdos para o público brasileiro não precisam só conhecer o palestrante estrangeiro, saber SI e ter experiência internacional para fazer uma boa interpretação. Conhecer e saber usar as estratégias de interpretação também é muito importante.

Mesmo que ainda não haja uma formação específica para interpretação de SI no Brasil, ainda é possível criar algumas demandas gerais, assim como a WASLI oferece para o mundo todo para quem deseja desenvolver a competência linguística, conhecimento situacional, e cultural para a interpretação intramodal - Língua de Sinais Brasileira para Sinais Internacionais, por exemplo, que tem sido vista recentemente nas conferências, pelos intérpretes surdos.

As pesquisas sobre intérpretes surdos no Brasil são ainda escassas; existem mais pesquisas internacionais sobre o assunto, desenvolvidas no mundo afora por pesquisadores e 
intérpretes experientes na área de tradução e/ou interpretação de língua de sinais. No Brasil, temos poucos intérpretes surdos, aproximadamente 26 (SILVEIRA, 2017). Alguns interpretaram apenas uma vez, outros são experientes e atuam como profissionais há anos, mas os intérpretes surdos em geral não têm formação profissional comprovada oficialmente e reconhecida como intérprete profissional e efetivo. No Decreto n. 5626 de 2005, o quarto capítulo discorre sobre a formação do tradutor e intérprete de Libras. Nota-se que não são apenas os intérpretes ouvintes que necessitam de formação superior, mas também os intérpretes surdos:

Profissional surdo, com competência para realizar a interpretação de língua de sinais de outros países para a Libras, para atuação em cursos e eventos (BRASIL, 2005).

É surpreendente que o bacharelado do curso de Letras-Libras inicialmente tenha sido criado exclusivamente para os ouvintes que querem se tornar intérpretes profissionais, como profissão oficialmente reconhecida com formação superior. Essa parte da lei demonstra a importância da inclusão dos intérpretes surdos no campo de tradução e/ou interpretação de Libras, que "sejam reconhecidos como intérprete profissional quanto aos intérpretes ouvintes de Língua de Sinais Brasileira" (REIS, 2013, p. 160). Então, devemos pensar sobre alterar o currículo do curso, incluir as novas disciplinas relacionadas aos estudos de SI, da interpretação intramodal e de outros assuntos relacionados aos intérpretes surdos, bem como criar os projetos para ampliar o conhecimento geral. Assim, eles terão oportunidade de aprender e praticar junto com os profissionais, adquirindo novos conhecimentos e ganhando novas experiências nessa área. A Federação Brasileira das Associações dos Profissionais Tradutores e Intérpretes e GuiaIntérpretes de Língua de Sinais (FEBRAPILS) ${ }^{38}$ também deve ampliar o reconhecimento dos tradutores, intérpretes e guia-intérpretes de língua de sinais (TILS), bem como os intérpretes surdos, e incluí-los no mercado do trabalho, atuando com a equipe dos intérpretes ouvintes.

No Brasil, então, o trabalho dos intérpretes surdos está no fim da segunda etapa do árduo caminho em direção ao reconhecimento da sua profissão. Durante a primeira etapa, foi preciso quebrar o tabu de que uma pessoa surda pode ser intérprete. Durante a segunda etapa, em conjunto com a inserção de pessoas surdas brasileiras em organizações internacionais de surdos, surgiram algumas pessoas com experiência em SI suficiente para atuarem pontualmente como intérpretes. Precisamos entrar na terceira etapa, de fomentar mais pesquisas na área e investir na qualificação e formação sistemática desses profissionais. 


\section{REFERÊNCIAS BIBLIOGRÁFICAS}

ADAM, R. Language contact and borrowing. In: PFAU, R.; STEINBACH, M.; WOLL, B. (Eds.). Sign language: An international handbook. Berlin: De Gruyter Mouton, 2012. p. 841861.

ADAM, R. et al. Deaf Interpreters at Work. Washington, DC: Gallaudet University, 2014.

BAKER-SHENK, C.; COKELY, D. American Sign Language. A Student Text Units 10-18. Washington, DC: Gallaudet University Press, 1991.

BOUDREAULT, P. Deaf Interpreters. In: JANZEN, T. (Ed.). Topics in signed language interpreting. Amsterdam: Benjamins, 2005. p. 323-356.

BRASIL. Decreto n. 5626, de 22 de dezembro de 2005. Regulamenta a Lei n. 10.436, de 24 de abril de 2002, que dispõe sobre a Língua Brasileira de Sinais - Libras, e o art. 18 da Lei n. 10.098, de 19 de dezembro de 2000. Disponível em:

http://www.planalto.gov.br/ccivil_03/_ato2004-2006/2005/decreto/d5626.htm. Acesso em: novembro de 2018.

BRITISH DEAF ASSOCIATION (BDA). Gestuno: International sign language of the deaf. The revised and enlarged book of signs agreed and adopted by the Unification of Signs

Commission of the World Federation of the Deaf. London: British Deaf Association, 1975.

BYUN, K. et al. First Encounters: Repair Sequences in Cross-Signing. Topics in Cognitive Science, p. 1-21, 2017.

CAMPELLO, A. R. e S. Intérprete surdo de língua de sinais brasileira: o novo campo de tradução / interpretação cultural e seu desafio. Cadernos de Tradução, Florianópolis, v. 1, n. 33, p. 143-167, jul. 2014. Disponível em:

https://periodicos.ufsc.br/index.php/traducao/article/view/2175-7968.2014v1n33p143. Acesso em: novembro de 2018.

CRASBORN, O.; HIDDINGA, A. Signed languages and globalization. Language in Society, Cambridge, p. 483-505, 2011.

FORESTAL, E. et al. Examples of a Deaf Interpreter's Work. Washington, DC: Gallaudet University, 2012. Disponível em: http://digitalcommons.unf.edu/asleimats/50/. Acesso em: novembro de 2018.

HANSEN, M. What Is International Sign? The Linguistic Status of a Visual Transborder Communication Mode. In: ROSENSTOCK, R.; NAPIER, J. (Eds.). International Sign: Linguistic, Usage, and Status Issues. Washington, DC: Gallaudet University Press, 2016. p. $15-31$.

HESSMANN, J. et al. Developing and transmitting a shared interpreting research ethos: EUMASLI - A case study. In: NICODEMUS, B.; SWABEY, L. (Eds.). Advances in Interpreting Resarch: Inquiry in Action. [S.1.]: Benjamins Translation Library, 2011. p. 177198. 
LUCHI, M. Interpretação de descrições imagéticas: onde está o léxico? Dissertação (Mestrado em Estudos da Tradução) - UFSC, Florianópolis, 2013.

MCKEE, R.; NAPIER, J. Interpreting into International Sign Pidgin: An analysis. Journal of Sign Language and Linguistics, p. 27-54, 2002.

MESCH, J. Perspectives on the Concept and Definition of International Sign. Helsinque: WFD, 2010. Disponível em: http://wfdeaf.org/wp-content/uploads/2016/11/Perspectives-onthe-Concept-and-Definition-of-IS_Mesch-FINAL.pdf. Acesso em: novembro de 2018.

MOODY, B. The role of International Sign Interpreting in today's world. In: ROY, C. B. (Ed.). Diversity and community in the worldwide Sign Language Interpreting

Profession: Proceedings of the second WASLI Conference, held in Segovia, Spain, 2007. [S.1.]: Douglas McLean, 2008. p. 19-33.

PEREIRA, M. C.P. Interpretação interlíngue: as especificidades da interpretação de língua de sinais. Cadernos de Tradução, Florianópolis, v. 1, p. 135-156, 2008.

PERLIN, G.; MIRANDA, W. Surdos: O narrar e a política. Ponto de Vista, Florianópolis, n. 5, p. 217-226, 2003.

REIS, F. O Surdo como intérprete. In: XII CONGRESSO INTERNACIONAL E XVIII SEMINÁRIO NACIONAL DO INES, 2013, Rio de Janeiro. Anais... Rio de Janeiro: INES, Departamento de Desenvolvimento Humano, Científico e Tecnológico, 2013.

RODRIGUES, C.; BEER, H. Os Estudos da Tradução e da Interpretação de Línguas de Sinais: Novo Campo Disciplinar Emergente? Cadernos da Tradução, Florianópolis, p. 17$45,2015$.

ROSENSTOCK, R.; NAPIER, J. Introduction: To International Sign or not to International Sign? That Is the Question. In: ___ International Sign: Linguistic, Usage and Status Issues. Whashington, DC: Gallaudet University Press, 2016. p. 1-12.

SILVEIRA, B. Intérprete Surdo: conquistando espaço no campo de conferências no Brasil. In: ALBRES, N. de A. (org.). Libras e sua tradução em pesquisa: interfaces, reflexões e metodologias. Florianópolis: Biblioteca Universitária UFSC, 2017. p. 14-37.

SHENEMAN, N.; COLLINS, P. F. The Complexities of Interpreting International Conferences: A Case Study. In: ROSENSTOCK, R.; NAPIER, J. (Eds.). International Sign: Linguistic, Usage and Status Issues. Whashington, DC: Gallaudet University Press, 2016. p. 167-191.

STONE, C. Toward a Deaf Translation Norm. Whashington, DC: Gallaudet University, 2009.

STONE, C.; RUSSELL, D. Interpreting in International Sign: Decisions of Deaf and NonDeaf Interpreters. In: CONFERENCE OF THE WORLD ASSOCIATION OF SIGN LANGUAGE INTERPRETERS (WASLI), 4., 2011, Durban, South Africa. Proceedings... Durban: WASLI, 2011. p. 100-118. 
SUPALLA, T.; WEBB, R. The grammar of International Sign: A new look at pidgin languages. In: EMMOREY, K.; REILLY, J. S. (Eds.). Language, gesture and space. Mahwah, NJ: Erlbaum, 1995.

WFD-WASLI International Sign Interpreter Accreditation Handbook. [S.1.]: WFD; WASLI, 2016. Disponível em: https://2tdzpf2t7hxmggqhq3njno1y-wpengine.netdna-ssl.com/wpcontent/uploads/2017/02/WFD-WASLI-IS-Interpreter-Accreditation_Handbook-31.8.pdf. Acesso em: novembro de 2018

WHYNOT, L. Telling Showing, and Representing: Conventions of the Lexicon in International Sign Expository Text. In: ROSENSTOCK, R.; NAPIER, J. (Eds.).

International Sign: Linguistic, Usage and Status Issues. Washington, DC: Gallaudet University Press, 2016. p. 35-64.

WIT, M. de; SLUIS, I. International Sign: An Exploration into Interpreter Preparation. In: ROSENSTOCK, R.; NAPIER, J. (Eds.). International Sign: Linguistic, Usage and Status Issues. Washington, DC: Gallaudet University Press, 2016. p. 105-135.

WORLD ASSOCIATION OF SIGN LANGUAGE INTERPRETERS (WASLI).

International Sign Definition. 2014. Disponível em: http://wasli.org/international-signdefinition. Acesso em: novembro de 2018.

ZESHAN, U. "Making meaning": Communication between sign language users without a shared language. Cognitive Linguistics, v. 26, n. 2, p. 211-260, 2015.

${ }^{\text {i }}$ Letícia Fernandes Garcia Wagatsuma GRANADO - Mestranda em Estudos da Tradução na Universidade Federal de Santa Catarina. Graduada em Letras/Libras (2016) pela mesma instituição. Florianópolis, Santa Catarina, Brasil. Lattes: http://lattes.cnpq.br/7969634634290242 E-mail: leticia.wagatsuma@gmail.com

${ }^{1}$ Todas as traduções são de minha autoria. International Sign (IS) is preferred because IS varies depending on the language background of the signers who use it.

${ }^{2}$ Use of the term International Sign, rather than International Sign Language, emphasises that IS is not recognised as having full linguistic status.

${ }^{3}$ We use the term INTERNATIONAL SIGN for the visual communication between deaf people from different countries who do not have a shared sign language.

${ }^{4}$ Sinalização cruzada.

${ }^{5}$ IS has been in use by European Deaf people for at least 250 years, but Deaf people from different countries in Europe were certainly meeting and successfully communicating with each other for a few hundred years before that.

${ }^{6}$ Surdolimpíadas, conhecida como Olimpíadas para Surdos, é um evento multidesportivo internacional.

${ }^{7}$ By the 1950s, the WFD felt that some standardization of 'international gestures' was desirable, and a committee on the 'unification of signs'.

${ }^{8}$ Naturally spontaneous and easy signs in common use by deaf people of different countries were selected. Disponível em: <http://brett-zamir.me/gestuno/?chapter=Introductory>. Acesso em: 2017.

${ }^{9}$ Deaf people soon began complaining that the signs in the Gestuno lexicon were not iconic enough to be readily understood. 
${ }^{10}$ The name Gestuno was chosen, referencing to gesture and a sense of oneness.

${ }^{11}$ The term Gestuno fell out of use, and so the book also fell out of favor.

${ }^{12}$ IS can be termed as kind of lingua franca used to overcome linguistic barriers.

${ }^{13}$ IS is surely not a kind of universal language understood in the same way and to the same extent by signers all over the world.

${ }^{14}$ Koines develop between spoken language where the languages in contact are extremely similar.

15 International Sign (IS) is an underresearched form of sign language contact that serves as a de facto lingua franca for globalizing deaf communities.

${ }^{16}$ It is not the native language of a Deaf community, transmitted from parents to children and peers to peers. It is used only in specific moments and in varying locations, when Deaf people with different native sign languages gather.

${ }^{17}$ International Sign is not an official language but is widely used at international meetings where participants do not share one common sign language.

${ }^{18}$ Fórum Europeu de Intérpretes de Língua de Sinais.

${ }^{19}$ União Europeia dos Surdos.

${ }^{20}$ Associação Mundial dos Intérpretes de Língua de Sinais.

${ }^{21}$ Federação Mundial de Surdos.

${ }^{22}$ Mestrado Europeu em Interpretação de Língua de Sinais.

${ }^{23}$ Associação Mundial de Intérpretes de Conferência.

${ }^{24}$ Federação Nacional de Educação e Integração dos Surdos, é uma entidade filantrópica, sem fins lucrativos, que tem por finalidade a defesa de políticas linguísticas, educação, cultura, saúde e assistência social, em favor da comunidade surda brasileira, bem como a defesa de seus direitos. Fonte: 〈http://feneis.org.br/sobre/>. Acesso em: 19 fev. 2018.

${ }^{25}$ The interpreter needs to have a variety of flexible interpreting strategies to respond to the unique demands in a multilingual and multicultural setting.

${ }^{26}$ First, as mentioned before, interpreters are usually trained in two languages and cultures. Working in international settings exposes interpreters to different levels of fluency in the various languages used at the event, [...] Next, additional complexities impact the interpreter's cognitive processing capacity as more effort is required. Third, the interpreter is tasked with working with non-native users of both languages that she is trained in.

${ }^{27}$ The definition of equivalence is central to the concept of free interpretation, and we contend that in the context of IS interpreting equivalence is sought by the interpreter inferring meaning based on their understanding of the situation and source language text and by predicting what the text is likely to mean to the target audience.

${ }^{28}$ ProLibras é um exame de proficiência para comprovar a fluência em Libras.

${ }^{29}$ How can a Deaf person be a signed language interpreter in your own Deaf community? It can't be. You're Deaf!

${ }^{30}$ A Deaf Interpreter is a specialist who provides interpreting, translation, and transliteration services in American Sign Language and other visual and tactual communication forms used by individuals who are Deaf, hard-ofhearing, and Deaf-Blind. Fonte: <http://www.diinstitute.org/what-is-the-deaf-interpreter/>. Acesso em: 17 mar. 2017.

${ }^{31}$ The professional use of Deaf Interpreters is increasing in several countries and across several contexts.

32 There are many possibilities for informal interpreting within Deaf community where some members of the community possess numerous skills to act as communication facilitators. The context can be within a Deaf school, the workplace or when meeting professional hearing people such as lawyers, doctors, etc. 
${ }^{33}$ how Deaf T/Is defined the job and how they defined an interpreter.

${ }^{34}$ These differences can identify whether the Deaf T/Is undertake the same job, a different job, or define the role of a "Deaf" interpreter as different from that of a "hearing" interpreter.

35 (1) Deaf and hearing interpreters are situated differently with respect to their habitus (Bourdieu, 1991) (i.e., where Deaf and hearing interpreters are culturally placed within a minority language community); (2) DIs and hearing interpreters treat nuances of language differently (e.g., DIS have a better understanding of sign language nuances, and hearing interpreters have a better understanding of spoken language nuances); (3) speech is not always a central part of a DI's work.

${ }^{36}$ Acceptance and recognition of the interpreter's language inventory, skills set, qualifications, and experience also differ between DI and hearing interpreters.

${ }^{37}$ INTERPRETER tends to represent hearing interpreters and DEAF IN-VISION represents Deaf T/Is.

38 A FEBRAPILS foi fundada em 22 de setembro de 2008, com o objetivo de orientar, apoiar e consolidar as Associações de Tradutores, Intérpretes e Guia-intérpretes de Língua de Sinais (APILS), buscando realizar um trabalho de parceria em defesa dos interesses da categoria de tradutores, intérpretes e guia-intérpretes de língua de sinais (TILS). 